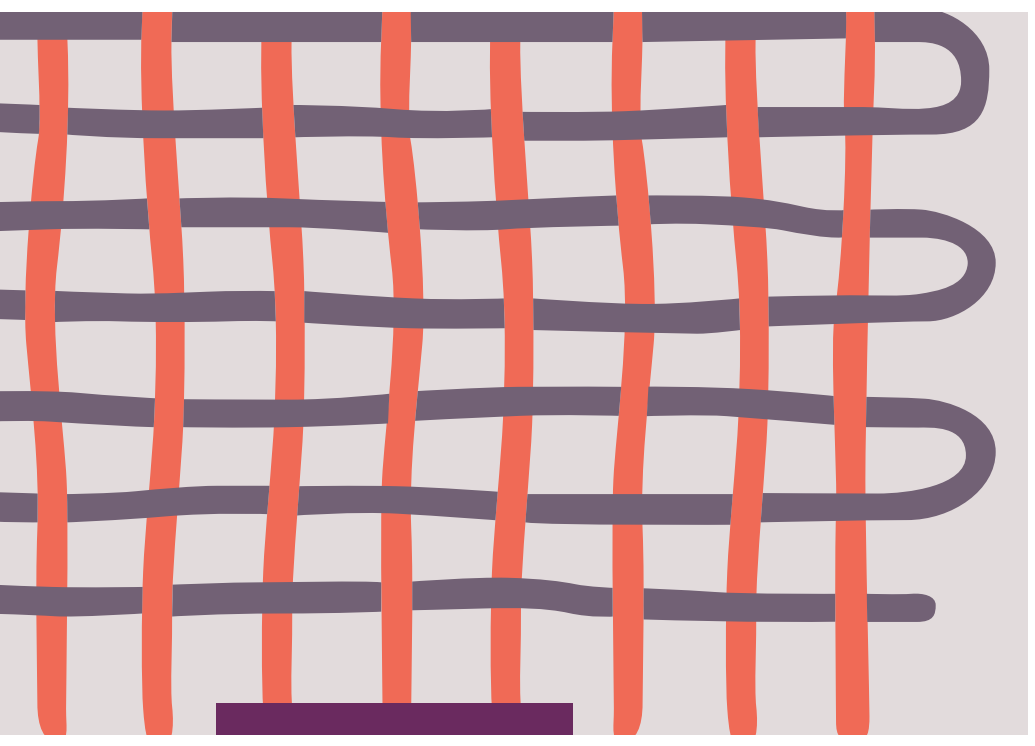

FIGURAS REVISTA ACADÉMICA DE INVESTIGACIÓN

ISSN 2683-2917

Vol. 1, núm. 3, julio-octubre 2020

https://doi.org/10.22201/fesa.figuras.2020.1.3

Esta obra está bajo una licencia

Creative Commons Atribución-NoComercial-

Compartirlgual 4.0 Internacional

\section{La urdimbre escolar. Una propuesta de investigación educativa}

\section{The school fabric. A proposal for educational research}

https://doi.org/10.22201/fesa.figuras.2020.1.3.122

Fernando Martínez-Vázquez

Universidad Nacional Autónoma de México.

Facultad de Estudios Superiores Acatlán. Colegio

de Ciencias y Humanidades Plantel Naucalpan

\section{Resumen}

El texto reseña los libros correspondientes al proyecto La urdimbre escolar: palabras y miradas. Se describe el contenido de cada uno y sus aportes al campo de la investigación educativa del nivel medio superior, particularmente se destacan investigaciones que han sido útiles para comprender los fenómenos sociales y culturales que se relacionan con alumnos y profesores, asimismo se describen líneas de investigación poco exploradas.

\section{Presentación}

La urdimbre escolar: palabras y miradas ${ }^{1}$ es un proyecto de investigación educativa que tiene como propósito analizar algunos procesos sociales y educativos que se presentan en la escuela; es producto de la necesidad de dar a conocer una parte de la cotidianidad que viven alumnos y profesores del Colegio de Ciencias y Humanidades ( $\mathrm{CCH}$ ) de la Universidad Nacional Autónoma de México (UNAM). Este proyecto tiene como objetivo generar líneas de investigación educativa desde el $\mathrm{CCH}$.

Toda propuesta es producto de las obsesiones, gustos, búsquedas, fantasías, miedos y dudas. En su libro Zona de obras, Leila Guerriero ${ }^{2}$ aporta múltiples ideas y recomendaciones para escribir, su consejo más contundente es tener algo que decir. A este proyecto lo precede una necesidad de expresar ideas, intereses, saberes, análisis, búsquedas, encuentros, desencuentros $\mathrm{y}$, sobre todo, preguntas acerca del cCH y de quienes lo habitan.

1 La Urdimbre Escolar es una propuesta de investigación educativa del y desde el $\mathrm{CCH}$, que se publica gracias al apoyo de la Dirección General de Asuntos del Personal Académico (DGAPA) de la Universidad Nacional Autónoma de México (UNAM), a través del proyecto PB:300616 "La urdimbre escolar: palabras y miradas", que forma parte de la Iniciativa para Fortalecer la Carrera Académica en el Bachillerato de la UNAM (INFOCAB).

2 Leila Guerriero, Zona de obras (México: Anagrama, 2014). 
Desarrollar el proyecto La urdimbre escolar: palabras y miradas implicó pensar en una institución con 49 años de existencia, reflexionar acerca del espacio en que han transitado miles de adolescentes y profesores con distintas características socio-culturales y económicas; analizar un espacio físico, social y simbólico en el que se han escrito historias, sedimentado recuerdos y vivido experiencias.

El proyecto se ha concretado en tres libros: La urdimbre escolar: palabras y miradas. Alumnos, ${ }^{3}$ Maestros fundadores ${ }^{4}$ y Caminos de la investigación. ${ }^{5}$ A continuación, se presenta una breve semblanza de cada uno.

\section{La escuela es un espacio de vida juvenil que no se limita a lo que sucede en el salón de clase. Más allá de lo académico, se sostiene en la sociabilidad o sentimiento y satisfacción de asociarse con otros.}

\section{Alumnos}

El primer libro, La urdimbre escolar: palabras y miradas. Alumnos, parte de concebir a la escuela como un espacio de interacción social en el cual se construyen vínculos emocionales, culturales, sociales, políticos y académicos, en el que -además- se comparten historias personales y colectivas.

3 Fernando Martínez Vázquez y Benita Areli Flores Martínez, coords., La urdimbre escolar: palabras y miradas. Alumnos (México: UNAM, 2017).

4 Fernando Martínez Vázquez, coord., La urdimbre escolar: palabras y miradas. Maestros fundadores (México: UNAM, 2018).

5 Fernando Martínez Vázquez, coord., La urdimbre escolar: palabras y miradas. Caminos de la investigación (México: UNAM, 2020).
En este libro se conceptualiza a los adolescentes como sujetos capaces de movilizarse social y simbólicamente, se les asume como actores en proceso de construcción. Se parte de la idea de que ser adolescente implica diversidad y complejidad, dependiendo de distintas variables culturales, económicas, sociales y de género.

Los adolescentes trasladan a la escuela sus trayectorias personales, construyen un nuevo tejido social, alternativo, complementario o en contraposición con el que experimentan en su diario vivir. La escuela es una prolongación de sus vidas, refugio o campo de batalla; lugar de definición, de continuidad y de ruptura.

Los alumnos encuentran en la escuela formas de interacción social que les permiten ratificarse y rectificarse: modos de estar juntos a través de prácticas sociales. La escuela adquiere distintos significados entre los que están:

- Obtener un certificado de bachillerato para ingresar a la educación superior.

- Cumplir con un requisito para ser contratados en algún empleo.

- Mostrarse que pueden desarrollarse, a sí mismos y a sus familias.

- Encontrarse con sus amigos, novios o parejas.

La escuela es un espacio de vida juvenil que no se limita a lo que sucede en el salón de clase, se extiende hacia los pasillos, en el relajo, juegos físicos, bromas, fiestas y reuniones. La escuela, más allá de lo académico, se sostiene en la sociabilidad o sentimiento y satisfacción de asociarse con otros. De acuerdo con lo que viven, se confrontan entre sus deseos y deberes con las instituciones.

El bachillerato de la UNAM y en particular el $\mathrm{CCH}$ pone a prueba la decisión de los adolescentes de vivir y experimentar la vida social en sus distintas dimensiones y riesgos; decidir si quieren cumplir con las tareas inherentes a su rol de estudiantes: entrar 
a clase, cumplir con actividades académicas, permanecer en la escuela, consumir o no drogas y alcohol.

Es también un espacio de vida juvenil en el que los alumnos transitan de manera no lineal: desarrollan recorridos educativos interrumpidos y fragmentados, tienen experiencias personales y sociales (construyen su identidad, tienen experiencias amorosas, sociales, culturales, reprueban materias, abandonan la escuela temporalmente y regresan). La experiencia escolar puede ser atractiva o no, los retiene o los expulsa hacia grupos de pares que se resisten al discurso institucional y se mantienen al margen desarrollando prácticas de riesgo. En el trayecto de los estudiantes hay momentos de decisión, tensión e incertidumbre donde no saben qué camino seguir: quedan a la deriva, toman tiempo para elegir e incluso, pueden seguir dudando.

Los alumnos del $\mathrm{CCH}$ viven un periodo de reconstrucción como actores sociales en busca de individualidad y autonomía, de nuevas adscripciones identitarias, con procesos de ruptura con lo vivido previamente, experimentando situaciones de rompimiento y liberación.

El libro se compone de los siguientes capítulos: "La necesidad de la investigación educativa en el $\mathrm{CCH}$ " de Enrique Pimentel Bautista; "La experiencia estudiantil de los jóvenes en el CCH" de Judith Adriana Díaz Rivera; "Telares reflexivos: Descubriendo CCH desde la mirada de quienes lo investigan" de María Guadalupe Sandoval Pantoja; "Trayectoria escolar y motivación de los jóvenes del turno vespertino del Colegio de Ciencias y Humanidades plantel Naucalpan" de Arlett Areli Ramírez Olguín y Miriam Rangel Paniagua; "Jóvenes y cсH: Construcción de experiencias y percepciones" de Iván Ruíz Salinas y Fabián Orlando Hernández Carranza; "Prácticas de riesgo en los estudiantes del cCH Naucalpan: Reporte de investigación" de Fernando Martínez Vázquez y Enrique Pimentel Bautista; "El CCH como espacio juvenil" de Mónica López Dorantes; "El fracaso escolar en los alumnos del Colegio de Ciencias y Humanida- des, plantel Sur. Estudio de caso" de Cinthia Reyes Jiménez y, por último, se presenta el texto "Una propuesta para hacer investigación de corte cualitativo y fenomenológico en el CCH", escrito por Ramón Cortés y Coronel y Roberto A. Zarate Córdoba.

La obra se complementa con retratos de los alumnos hechos por Leonardo Pliego Eguiluz - extraordinario ejercicio fotográfico que muestra las personalidades de los estudiantes del $\mathrm{CCH}-\mathrm{y}$ su texto Incontenibles.

\section{Maestros fundadores}

El Colegio de Ciencias y Humanidades está a punto de cumplir 50 años. Desde su fundación, el país, la Universidad y la institución se han trasformado. La sociedad actual atraviesa por una crisis económica, social, política y educativa que hace urgente reflexionar acerca del origen y sentido del CCH y su modelo educativo. En este contexto se consideró necesario otorgar la palabra a quienes construyeron al Colegio desde el salón de clases: los profesores. El libro La urdimbre escolar: palabras y miradas. Maestros fundadores recupera sus experiencias, el cómo, por qué y para qué de ser docente.

En él se presentan las historias de profesoras y profesores que edificaron el $\mathrm{CCH}$ desde sus primeros días. Se partió de tres preguntas: ¿Cómo? ¿Por qué? y ¿Para qué ser docente?, se exploraron los aspectos personales de los docentes tratando de recuperar su visión de la vida, de la docencia y de sí mismos.

Para esta investigación se entrevistaron 16 maestros en activo, fundadores del $\mathrm{CCH}$ Naucalpan. Entendiendo por fundadores a quienes empezaron a dar clase entre 1971 y 1973. Los maestros entrevistados son: Marisela Álvarez Pérez, Vicente Ballesteros Linares, José de Jesús Bazán Levy, Juan Antonio Flores Lira, Marco Antonio Lagarde Torres, José Alfredo del Sagrado Corazón de Jesús Martínez y Arronte, Miguel Mercado Martínez, Lilia Olivia Muñoz Barrueta, Rosa Elba Pérez Orta, Susana Ramírez Ruiz 
Esparza, Juan Javier de San José Ramírez, Cirilo Sánchez Vargas, Piedad Solís Mendoza y Lorenzo Manuel Vega Suárez.

El texto se compone de tres partes: la primera expone una contextualización de la investigación cualitativa y la pertinencia de la entrevista a profundidad, así como un estado de la cuestión de la investigación educativa acerca de profesores; la segunda presenta las entrevistas a manera de semblanzas; la tercera está compuesta por los retratos de Leonardo Pliego Eguiluz que, a través de la imagen, dan cuenta de la personalidad de quienes fundaron el $\mathrm{CCH}$.

\section{Los profesores son personajes centrales en todo proceso educativo. Son quienes construyen las instituciones. Los alumnos se van, los profesores permanecen.}

Luz y sombra, los profesores son personajes centrales en todo proceso educativo. Son quienes construyen las instituciones, las fortalecen o debilitan, hacen de ellas espacios vivos. Los alumnos se van, los profesores permanecen, constituyen la estructura, la base humana de los procesos educativos.

Ser profesor implica ejercer un oficio de lo humano, una práctica profesional que se sustenta en la relación con el otro y la complejidad que esto implica. Una relación que se constituye a través de un proceso de enseñanza y aprendizaje, de la experiencia subjetiva, de las formas en que nos apropiamos de lo vivido, son representaciones a las que otorgamos armonía. Todo adquiere una dimensión personal, la hacemos parte de nuestra historia y de cómo la contamos.

En el libro se recupera la memoria personal de los profesores, lo cual permitió conocer de dónde vienen, la importancia de su familia, así como el contexto vi- vido y recordado. Se recopilan recuerdos, anécdotas, imágenes que entretejen los escenarios del pasado. En las distintas historias, la memoria subjetiva es eco del pasado y ventana del futuro.

El tiempo es la categoría de la experiencia humana. El tiempo está ligado a la historia natural, social y personal; la memoria se construye desde el lapso en que se reflexiona y desde el momento de la existencia. El tiempo sólo se puede observar a partir de uno mismo, de nuestra condición personal y subjetiva. Nosotros pasamos: somos tiempo y el tiempo nos constituye. De esto y más habla el segundo libro La urdimbre escolar: palabras y miradas. Maestros Fundadores.

\section{Caminos de la investigación}

El tercer libro, La urdimbre escolar: palabras y miradas. Caminos de la investigación, tuvo como propósito explorar líneas de investigación poco tratadas en el campo de la educación, como es el caso del porrismo, el enfoque de género, la percepción del cuerpo y la identidad, las trayectorias escolares, la identidad docente, la enseñanza de la ciudadanía, el rezago escolar y los métodos de intervención.

En este último libro se trabajan de forma independiente diversos artículos que aportan reflexiones, datos y análisis de los temas referidos de manera tal que se plantean nuevas líneas de investigación posibles y que se podrían explorar en el futuro.

La primera parte inicia con el texto "Construcción de la identidad dentro de organizaciones estudiantiles de violencia institucionalizada: porros del $\mathrm{CCH}$ Naucalpan", de José Luis Alvarado Sánchez y Karen Gabriela Lozada González quienes, por medio de trabajo de campo y entrevistas, recuperan una serie de elementos para analizar e interpretar las dinámicas, razones y motivos que mueven a actuar a estos grupos de jóvenes.

A continuación se presenta "La corporalidad juvenil en el cсH. Percepciones sobre las clases sociales, 
la estética y las características comunicativas del cuerpo" de los autores Alan Jesús Cruz Acosta, Wenceslao Melgarejo Ramos, Eduardo Abraham Montero Soster y Mónica Rubalcava Loredo, quienes exponen las construcciones simbólicas del grupo de adolescentes del CCH Naucalpan. Este texto aborda un objeto de estudio poco explorado en el campo educativo que es el cuerpo y su relación con las representaciones sociales y la identidad.

Por su parte, Judith Adriana Díaz Rivera analiza las diferencias académicas entre hombres y mujeres en el nivel medio superior, a través de la descripción y análisis de las trayectorias educativas de los alumnos del cCH. Su texto "Algunas diferencias en las trayectorias educativas de las y los estudiantes en el Colegio de Ciencias y Humanidades" plantea la necesidad de abordar el desempeño escolar desde una perspectiva de género e implementar estrategias de apoyo diferenciadas para promover la permanencia y egreso del bachillerato de mujeres y hombres.

Hay investigaciones sobre el tránsito de la educación básica a la media superior pero se enfocan en los resultados y no en los procesos que los alumnos siguen
en sus trayectorias.

Las trayectorias de la educación básica a la educación media superior es el objeto de referencia del texto "El estudio del tránsito de la educación básica a la educación media superior: una crítica metodológica". En él, Alberto Tonatiuh Alonso Sarmiento plantea que la mayoría de las investigaciones que tratan este tema se enfocan en los resultados y no en el tránsito entre un nivel y otro, lo que ha impedido que se profundice en los procesos que siguen los alumnos en sus trayectorias.
Fernando Martínez Vázquez, Enrique Pimentel Bautista y René Cuellar Serrano presentan "La urdimbre escolar. Una mirada a los alumnos del turno vespertino del CCH Naucalpan, UNAM", texto que explica los resultados de tres investigaciones realizadas con estudiantes del turno vespertino en las que se analizan las percepciones que tienen acerca de sus problemáticas, asociadas a lo juvenil, sus prácticas culturales, roles sexuales, así como su concepción del entorno que los rodea.

Un trabajo interesante porque muestra una estrategia de mediación, es "Los factores de rezago escolar en el CCH Plantel Vallejo. Una actividad de intervención educativa" co-autoría de los profesores Ramón Cortés y Coronel y Roberto Alfredo Zárate Córdova, quienes proponen una actividad de intervención educativa con alumnos de la asignatura Taller de Lectura, Redacción e Iniciación a la Investigación Documental. Dicha estrategia recurre a técnicas teatrales basadas en el método Stanislavsky a través del cual logran que alumnos con problemas de aprendizaje desarrollen habilidades comunicativas y confianza en sí mismos.

La profesora Rocío Valdés Quintero comparte una reflexión acerca del significado de educar en la época contemporánea para formar ciudadanos en el marco del aprendizaje de valores en su texto "Cómo educar en valores en el marco del modelo educativo del $\mathrm{CCH}$ "; para ello, toma como referencia el principio pedagógico de "Aprender a ser", con el propósito de analizar cómo construir comunidades para fomentar en los estudiantes una conciencia democrática capaz de contribuir a la formación de un razonamiento crítico y sentido de responsabilidad en la vida social.

La segunda parte del libro está dedicada a los profesores, inicia con el texto de Fernando Martínez Vázquez e Iriana González Mercado, "Comunicación, memoria e identidad. El caso del CCH, UNAM, México", en el cual rescatan la noción de docencia producto del análisis de las narrativas de las trayectorias personales de profesores con trayectorias de 47 años, 
con el objetivo de explicar la función de la comunicación en los procesos de reconstrucción de la memoria e identidad docente.

Cinthia Reyes Jiménez en "El docente de hoy", aborda el tema de ser profesor desde diferentes miradas críticas, que parten de las transformaciones que se han gestado en el sistema educativo mexicano, y que considera elementos políticos, económicos y culturales de la sociedad.

La tercera parte del libro contiene la mirada artística de Leonardo Eguiluz con "Las personas pueden volar. Jóvenes, tatuajes, suspensiones y modificaciones corporales. Ensayo fotográfico", para lo cual se fotografió una muestra de una mirada distinta acerca del imaginario social que ha caracterizado al tatuaje y las modificaciones corporales. La serie de fotografías muestran la autorepresentación de un grupo de jóvenes en la Ciudad de México y brinda un panorama particular de la juventud. Con este material visual se motivan reflexiones acerca del concepto de belleza y las prácticas de discriminación.

La urdimbre escolar es un proyecto académico de investigación educativa que busca aportar elementos y reflexiones acerca de la escuela como lugar social total y de los actores que la habitan en la educación media superior. A través de sus páginas se pueden encontrar diversas miradas del fenómeno educativo y su complejidad. Es un aporte para reflexionar acerca de la educación media superior, sus diversos aspectos y problemáticas, pero además ha sido un punto de encuentros de académicos e investigadores de la UNAM y de otras instituciones, quienes han compartido sus miradas al fenómeno de la educación a través de las páginas que conforman estos libros.

Los libros del proyecto La urdimbre escolar: palabras y miradas se han presentado en la Feria Internacional del Libro del Palacio de Minería 2017, 2018 y 2020, en los Congresos Nacionales de Investigación Educativa 2017 y 2019; en la Facultad de Estudios Superiores Acatlán, UNAM 2020 y los planteles Naucalpan, Sur y Vallejo del CCH, así como en la Cátedra UNESCO en la ciudad de Bogotá Colombia en el año de 2018.

\section{Referencias}

Guerrero Salinas, M. Elsa. "Algunos rasgos de la experiencia estudiantil en el bachillerato." En Jóvenes y bachillerato. Coordinado por Eduardo Weiss, 125-150. México: ANUIES, 2012.

Guerriero, Leila. Zona de obras. México: Anagrama, 2014. Martínez Vázquez, Fernando y Flores Martínez Benita Areli, coords. La urdimbre escolar: palabras y miradas. Alumnos. México: UNAM, 2017.

Martínez Vázquez, Fernando, coord. La urdimbre escolar: palabras y miradas. Caminos de la investigación. México: UNAM, 2020.

Martínez Vázquez, Fernando, coord. La urdimbre escolar: palabras y miradas. Maestros fundadores. México: UNAM, 2018.

Weiss, Eduardo, coord. Jóvenes y bachillerato. México: ANUIES, 2012. 